PROCEEDINGS OF THE

AMERICAN MATHEMATICAL SOCIETY

Volume 135, Number 6, June 2007, Pages 1695-1699

S 0002-9939(06)08632-1

Article electronically published on November 29, 2006

\title{
ALGEBRAIC REFLEXIVITY OF LINEAR TRANSFORMATIONS
}

\author{
JIANKUI LI AND ZHIDONG PAN
}

(Communicated by Joseph A. Ball)

\begin{abstract}
Let $\mathcal{L}(U, V)$ be the set of all linear transformations from $U$ to $V$, where $U$ and $V$ are vector spaces over a field $\mathbb{F}$. We show that every $n$ dimensional subspace of $\mathcal{L}(U, V)$ is algebraically $\lfloor\sqrt{2 n}\rfloor$-reflexive, where $\lfloor t\rfloor$ denotes the largest integer not exceeding $t$, provided $n$ is less than the cardinality of $\mathbb{F}$.
\end{abstract}

\section{INTRODUCTION}

Let $U$ and $V$ be vector spaces over a field $\mathbb{F}$, let $\mathcal{L}(U, V)$ be the set of all linear transformations from $U$ to $V$, and let $\mathcal{L}_{F}(U, V)$ be the set of finite rank transformations in $\mathcal{L}(U, V)$. For any $x \in U$ and subspace $\mathcal{S} \subseteq \mathcal{L}(U, V)$, let $\mathcal{S} x=\{A x: A \in \mathcal{S}\}$. Define $\operatorname{ref}_{a}(\mathcal{S})=\{T \in \mathcal{L}(U, V): T x \in \mathcal{S} x$, for all $x \in U\}$. $\mathcal{S}$ is called algebraically reflexive if $\operatorname{ref}_{a}(\mathcal{S})=\mathcal{S}$. Define $\mathcal{S}^{(n)}=\left\{S^{(n)} \in \mathcal{L}\left(U^{(n)}, V^{(n)}\right): S \in \mathcal{S}\right\}$, where $U^{(n)}$ is the direct sum of $n$ copies of $U, V^{(n)}$ is the direct sum of $n$ copies of $V$, and $S^{(n)}$ is the direct sum of $n$ copies of $S$. $\mathcal{S}$ is called algebraically $n$-reflexive if $\mathcal{S}^{(n)}$ is algebraically reflexive in $\mathcal{L}\left(U^{(n)}, V^{(n)}\right)$. Clearly, if $\mathcal{S}$ is algebraically $n$ reflexive and $n<m$, then $\mathcal{S}$ is algebraically $m$-reflexive. A vector $x \in U$ is called a separating vector of $\mathcal{S}$ if the evaluation map $E_{x}: A \rightarrow A x, A \in \mathcal{S}$ is injective. It is well known and easy to prove that if $\mathcal{S}$ has a separating vector, then it is algebraically 2-reflexive. The local dimension of $\mathcal{S}$, denoted by $k(\mathcal{S})$, is defined by $k(\mathcal{S})=\max \{\operatorname{dim}(\mathcal{S} x): x \in U\}$; clearly $k(\mathcal{S}) \leq \operatorname{dim} \mathcal{S}$. If $\operatorname{dim} \mathcal{S}<\infty$, then $k(\mathcal{S})=\operatorname{dim} \mathcal{S}$ if and only if $\mathcal{S}$ has a separating vector. Thus, the concept of local dimension generalizes that of separating vectors.

If $U=V=H$ are complex Hilbert spaces and $\mathcal{S}$ is a finite-dimensional subspace of $B(H)$, where $B(H)$ denotes the set of all bounded linear operators, then algebraic reflexivity coincides with the reflexivity of $\mathcal{S}$. (For the definition and general results on reflexivity of subspaces of operators, [1] and [3] are excellent references.) The main result in 6 states that if $\mathcal{S}$ is an $n$-dimensional subspace of $B(H)$, where $H$ is a separable complex Hilbert space, then $\mathcal{S}$ is algebraically $\lfloor\sqrt{2 n}\rfloor$-reflexive.

While the above result answered a question raised in [7, the proof given in [6] is quite technical and relies on results from operator theory on Hilbert spaces. The main purpose of this paper is to generalize the above result to linear transformations on vector spaces and provide a simpler and more self-contained proof.

Received by the editors August 21, 2005 and, in revised form, January 5, 2006.

2000 Mathematics Subject Classification. Primary 47L05; Secondary 15A04.

Key words and phrases. Algebraic reflexivity, separating vector.

This research was partially supported by the NSF of China.

(C)2006 American Mathematical Society 
Let $|\mathbb{F}|$ denote the cardinality of $\mathbb{F}$. The main result of the paper is the following.

Theorem 1. If $\mathcal{S}$ is an $n$-dimensional subspace of $\mathcal{L}(U, V)$ with $n<|\mathbb{F}|$, then $\mathcal{S}$ is algebraically $\lfloor\sqrt{2 n}\rfloor$-reflexive.

The notion of algebraic reflexivity was first introduced in [4]. In [5], the following well-known result on algebraic reflexivity is proved: If $\mathcal{S}$ is a finite-dimensional subspace of $\mathcal{L}(U, V)$, then $\operatorname{ref}_{a}(\mathcal{S})=\mathcal{S}+\operatorname{ref}_{a}\left(\mathcal{S} \cap \mathcal{L}_{F}(U, V)\right)$, where $\mathcal{L}_{F}(U, V)$ denotes the set of all finite rank linear transformations in $\mathcal{L}(U, V)$. It follows that $\mathcal{S}$ is algebraically $n$-reflexive if and only if $\mathcal{S} \cap \mathcal{L}_{F}(U, V)$ is algebraically $n$-reflexive; so, for the remainder of the paper, we may assume that $\mathcal{S}$ is a subspace of $\mathcal{L}(U, V)$, where $U$ and $V$ are finite-dimensional vector spaces over $\mathbb{F}$.

The following is essentially the same as [2, Proposition 1.1].

Lemma 2 [[2]]. Suppose $\mathcal{S}$ is an $n$-dimensional subspace of $\mathcal{L}(U, V)$ with $n<|\mathbb{F}|, x$ is a separating vector of $\mathcal{S}$, and $W$ is a linear subspace of $V$ satisfying $\mathcal{S} x \cap W=(0)$. Then for each vector $y \in U$, with the exception of at most $n$ values of $\lambda \in \mathbb{F}, y+\lambda x$ separates $\mathcal{S}$ and $\mathcal{S}(y+\lambda x) \cap W=(0)$.

Let $M$ be a subspace of $V$. For $T \in \mathcal{L}(U, V)$, let $R(T)$ denote the range of $T$. Define $\mathcal{S}_{M}=\{A \in \mathcal{S}: R(A) \subseteq M\}$. Let $P_{M^{c}}$ be the projection of $V$ onto any vector space complement of $M$ in $V$ (the projection is done through $M$ ) and let $\mathcal{S}_{M}^{c}$ be any vector space complement of $\mathcal{S}_{M}$ in $\mathcal{S}$. Define $P_{M^{c}} \mathcal{S}_{M}^{c}=\left\{P_{M^{c}} A: A \in \mathcal{S}_{M}^{c}\right\}$.

Lemma 3. If $\mathcal{S}$ is an $n$-dimensional subspace of $\mathcal{L}(U, V)$ with $n<|\mathbb{F}|$, then $k\left(\mathcal{S}_{M}\right)+$ $k\left(P_{M^{c}} \mathcal{S}_{M}^{c}\right) \leq k(\mathcal{S})$.

Proof. If $P_{M^{c}} \mathcal{S}_{M}^{c}=\{0\}$, then clearly $k\left(\mathcal{S}_{M}\right) \leq k(\mathcal{S})$.

If $\mathcal{S}_{M}=\{0\}$, then $k\left(P_{M^{c}} \mathcal{S}_{M}^{c}\right) \leq k\left(\mathcal{S}_{M}^{c}\right) \leq k(\mathcal{S})$.

Suppose $k\left(\mathcal{S}_{M}\right)=m \neq 0$ and $k\left(P^{\perp} \mathcal{S}_{M}^{c}\right)=l \neq 0$. Let $\left\{A_{1}, \ldots, A_{m}\right\} \subseteq \mathcal{S}_{M}$ be linearly independent and let $x_{0} \in U$ be a separating vector of $\operatorname{span}\left\{A_{1}, \ldots, A_{m}\right\}$. Suppose $\left\{B_{1}, \ldots, B_{l}\right\} \subseteq \mathcal{S}_{M}^{c}$ so that $P_{M^{c}} B_{1}, \ldots, P_{M^{c}} B_{l}$ are linearly independent and $y_{0}$ is a separating vector of $\operatorname{span}\left\{P_{M^{c}} B_{1}, \ldots, P_{M^{c}} B_{l}\right\}$.

First, we show that $\operatorname{span}\left\{A_{1}, \ldots, A_{m}\right\}$ and $\operatorname{span}\left\{P_{M^{c}} B_{1}, \ldots, P_{M^{c}} B_{l}\right\}$ have a common separating vector: by Lemma 2 , there are at most $l$ values of $\lambda \in \mathbb{F}$ such that $x_{0}+\lambda y_{0}$ are not separating vectors of $\operatorname{span}\left\{P_{M^{c}} B_{1}, \ldots, P_{M^{c}} B_{l}\right\}$. We can assume 0 is one of those values; otherwise, $x_{0}$ is a common separating vector. Among the other values in $\mathbb{F}$, by Lemma 2 again, there are at most $m$ values $\lambda$ such that $x_{0}+\lambda y_{0}$ are not separating vectors of $\operatorname{span}\left\{A_{1}, \ldots, A_{m}\right\}$; otherwise, there would be more than $m$ vectors of the form $\lambda^{-1} x_{0}+y_{0}$ that are not separating vectors of $\operatorname{span}\left\{A_{1}, \ldots, A_{m}\right\}$. Since $m+l \leq n<|\mathbb{F}|$, there exists a $\lambda_{0}$ such that $x_{0}+\lambda_{0} y_{0}$ is a common separating vector.

Let $z_{0}=x_{0}+\lambda_{0} y_{0}$. It remains to show that $A_{1} z_{0}, \ldots, A_{m} z_{0}, B_{1} z_{0}, \ldots, B_{l} z_{0}$ are linearly independent.

For any $\lambda_{1}, \ldots, \lambda_{m}, \mu_{1}, \ldots, \mu_{l} \in \mathbb{F}$, suppose

$$
\lambda_{1} A_{1} z_{0}+\ldots+\lambda_{m} A_{m} z_{0}+\mu_{1} B_{1} z_{0}+\ldots+\mu_{l} B_{l} z_{0}=0 .
$$

Applying $P_{M^{c}}$ to both sides of (1), it follows that

$$
\mu_{1} P_{M^{c}} B_{1} z_{0}+\ldots+\mu_{l} P_{M^{c}} B_{l} z_{0}=0 .
$$

Since $z_{0}$ is a separating vector of $\operatorname{span}\left\{P_{M^{c}} B_{1}, \ldots, P_{M^{c}} B_{l}\right\}$, we have $\mu_{1}=\ldots=\mu_{l}=$ 0 . Now equation (1) implies $\lambda_{1}=\ldots=\lambda_{m}=0$, since $z_{0}$ is a separating vector of $\operatorname{span}\left\{A_{1}, \ldots, A_{m}\right\}$. Hence $k(\mathcal{S}) \geq k\left(\mathcal{S}_{M}\right)+k\left(P_{M^{c}} \mathcal{S}_{M}^{c}\right)$. 
Lemma 4. Suppose $\mathcal{S}$ is an $n$-dimensional subspace of $\mathcal{L}(U, V)$ with $n<|\mathbb{F}|$ and $k(\mathcal{S})=k$. For $x_{0} \in U$ and $A_{1}, \ldots, A_{k} \in \mathcal{S}$, let $M=\operatorname{span}\left\{A_{1} x_{0}, \ldots, A_{k} x_{0}\right\}$. If $\operatorname{dim} M=k$, then $\mathcal{S}_{M}$ contains all $T \in \mathcal{S}$ with $T x_{0}=0 ;$ moreover, $\operatorname{dim}\left(\mathcal{S}_{M}^{c}\right) \leq k$.

Proof. To prove the first part, we proceed contrapositively. Assume there exist $T \in$ $\mathcal{S}$ with $T x_{0}=0$ and $y \in U$ such that $T y \notin \operatorname{span}\left\{A_{1} x_{0}, \ldots, A_{k} x_{0}\right\}$; then necessarily $T \neq 0$. Let $W$ be the one-dimensional subspace $(\mathbb{F} T y)$ and $\widehat{\mathcal{S}}=\operatorname{span}\left\{A_{1}, \ldots, A_{k}\right\}$. Then $\widehat{\mathcal{S}} x_{0} \cap W=(0)$. By Lemma 2 , there exists $\lambda \in \mathbb{F}$ such that $y+\lambda x_{0}$ separates $\widehat{\mathcal{S}}$ and $\widehat{\mathcal{S}}\left(y+\lambda x_{0}\right) \cap W=(0)$. Since $T x_{0}=0$, it follows that $\left\{A_{1}, \ldots, A_{k}, T\right\}$ is linearly independent. Let $\widetilde{\mathcal{S}}=\operatorname{span}\left\{A_{1}, \ldots, A_{k}, T\right\}$. Next we prove that $y+\lambda x_{0}$ separates $\widetilde{\mathcal{S}}$. For any $A \in \widehat{\mathcal{S}}, t \in \mathbb{F}$, if $(A+t T)\left(y+\lambda x_{0}\right)=0$, then $A\left(y+\lambda x_{0}\right)=-t T y$. By $\widehat{\mathcal{S}}\left(y+\lambda x_{0}\right) \cap W=(0)$, it follows that $t=0$ and $A\left(y+\lambda x_{0}\right)=0$. Since $y+\lambda x_{0}$ is a separating vector of $\widehat{\mathcal{S}}$, we have $A=0$. Hence $y+\lambda x_{0}$ separates $\widetilde{\mathcal{S}}$, which implies $k(\mathcal{S}) \geq k+1$, a contradiction.

To see the second part, take any $B \in \mathcal{S}$; since $M=\mathcal{S} x_{0}$, there exist $a_{1}, \ldots, a_{k} \in \mathbb{F}$ such that $B x_{0}=a_{1} A_{1} x_{0}+\ldots+a_{k} A_{k} x_{0}$. By the first part, $B-\left(a_{1} A_{1}+\ldots+a_{k} A_{k}\right) \in$ $\mathcal{S}_{M}$

For any $d$-dimensional vector space $W$ over a field $\mathbb{F}$, let $W^{*}$ be the space of linear functionals on $W$. Note that both $W$ and $W^{*}$ can be identified naturally with $\mathbb{F}^{(d)}$. We can view $W$ as $W^{* *}$ as well. For any $T \in \mathcal{L}(U, V)$, define $T^{*} \in \mathcal{L}\left(V^{*}, U^{*}\right)$ as follows: For any $f \in V^{*}, T^{*} f$ is defined by $\left(T^{*} f\right)(u)=f(T u), \forall u \in U$. Letting $\mathcal{S}^{*}=\left\{A^{*}: A \in \mathcal{S}\right\}$, we have

Lemma 5. $\mathcal{S}$ is algebraically reflexive in $\mathcal{L}(U, V)$ if and only if $\mathcal{S}^{*}$ is algebraically reflexive in $\mathcal{L}\left(V^{*}, U^{*}\right)$.

Proof. An application of a version of the Hahn-Banach separation theorem yields:

$T \in \operatorname{ref}_{a}(\mathcal{S}) \Longleftrightarrow T u \in \mathcal{S} u, \forall u \in U$

$\Longleftrightarrow f(T u) \in\{f(A u): \forall A \in \mathcal{S}\}, \forall u \in U, \forall f \in V^{*}$

$\Longleftrightarrow\left(T^{*} f\right)(u) \in\left\{\left(A^{*} f\right)(u): \forall A \in \mathcal{S}\right\}, \forall u \in U, \forall f \in V^{*}$

$\Longleftrightarrow T^{*} f \in\left\{A^{*} f: \forall A \in \mathcal{S}\right\}, \forall f \in V^{*}$

$\Longleftrightarrow T^{*} \in \operatorname{ref} f_{a}\left(\mathcal{S}^{*}\right)$.

If $U$ is a $k$-dimensional vector space, then every subspace of $\mathcal{L}(U, V)$ is algebraically $k$-reflexive. This follows from the fact that two linear transformations agreeing on a basis of $U$ must be identical.

Lemma 6. If $\operatorname{dim}(\operatorname{ran}(\mathcal{S}))=k$, then $\mathcal{S}$ is algebraically $k$-reflexive.

Proof. Let $V_{1}=\operatorname{ran}(\mathcal{S})$. Without loss of generality, we assume $\mathcal{S} \subseteq \mathcal{L}\left(U, V_{1}\right)$. Since $\operatorname{dim} V_{1}=k, \mathcal{S}^{*}$ is algebraically $k$-reflexive. Hence $\mathcal{S}$ is algebraically $k$-reflexive by Lemma 5 .

Lemma 7. Suppose $\mathcal{S}$ is an $n$-dimensional subspace of $\mathcal{L}(U, V)$ with $n<|\mathbb{F}|$. If $k(\mathcal{S})=k$, then $\mathcal{S}$ is algebraically $k$-reflexive.

Proof. We will proceed by induction on $k$.

First suppose $k=1$ and take any $T \in \operatorname{ref} f_{a}(\mathcal{S})$. Let $\widetilde{\mathcal{S}}=\operatorname{span}\{T, \mathcal{S}\}$. Suppose $\operatorname{dim}(\widetilde{\mathcal{S}}) \geq 2$ (otherwise, there is nothing to prove). Since $T \in \operatorname{ref} f_{a}(\mathcal{S}), k(\widetilde{\mathcal{S}})=1$. By Lemma 4 , there exists a subspace $M$ of $V$ with $\operatorname{dim} M=1$ such that $\operatorname{dim}(\widetilde{\mathcal{S}})_{M}^{c} \leq 1$. Since $k(\widetilde{\mathcal{S}})=1,(\widetilde{\mathcal{S}})_{M}^{c}=(0)$ by Lemma 3 ; i.e., $\operatorname{dim}(\operatorname{ran}(\widetilde{\mathcal{S}}))=1$. In particular, $\operatorname{dim}(\operatorname{ran}(\mathcal{S}))=1$. Therefore, $\mathcal{S}$ is algebraically reflexive by Lemma 6 . 
Suppose the statement is true for all $\mathcal{S}$ with $k(\mathcal{S}) \leq k-1$, where $k \geq 2$.

For any $\mathcal{S}$ with $k(\mathcal{S})=k$, where $k \geq 2$, there exist $x_{1} \in U$ and $\left\{A_{1}, \ldots, A_{k}\right\} \subseteq \mathcal{S}$ such that $\left\{A_{i} x_{1}\right\}_{i=1}^{k}$ is a basis of $\mathcal{S} x_{1}$. Suppose $\mathcal{S}=\operatorname{span}\left\{A_{1}, \ldots, A_{n}\right\}$. There exists a unique $k \times n$ matrix $\left(a_{i j}\right)$ with $a_{i j} \in \mathbb{F}$ such that

$$
A_{j} x_{1}=\sum_{i=1}^{k} a_{i j} A_{i} x_{1}, j=1, \ldots, n,
$$

where if $j \leq k, a_{j j}=1$ and $a_{i j}=0$ for $i \neq j$.

It is not hard to see that any linear transformation in $\operatorname{ref}_{a}\left(\mathcal{S}^{(k)}\right)$ must be of the form $T^{(k)}$, for some $T \in \mathcal{L}(U, V)$. Given any $T^{(k)} \in \operatorname{ref} f_{a}\left(\mathcal{S}^{(k)}\right)$, then for any $x_{2}, \ldots, x_{k} \in U$, there exist $t_{1}, \ldots, t_{n} \in \mathbb{F}$ such that

$$
\left(\begin{array}{c}
T x_{1} \\
\vdots \\
T x_{k}
\end{array}\right)=t_{1}\left(\begin{array}{c}
A_{1} x_{1} \\
\vdots \\
A_{1} x_{k}
\end{array}\right)+\ldots+t_{n}\left(\begin{array}{c}
A_{n} x_{1} \\
\vdots \\
A_{n} x_{k}
\end{array}\right) .
$$

Since $T x_{1} \in \operatorname{span}\left\{A_{1} x_{1}, \ldots, A_{k} x_{1}\right\}$, there exist unique fixed $c_{1}, \ldots, c_{k} \in \mathbb{F}$ such that

$$
T x_{1}=\sum_{i=1}^{k} c_{i} A_{i} x_{1}
$$

By (4) and (5), we have

$$
T x_{m}=\sum_{i=1}^{k} c_{i} A_{i} x_{m}+\sum_{j=1}^{n} t_{j}\left(A_{j}-\sum_{i=1}^{k} a_{i j} A_{i}\right) x_{m}, m=2, \ldots, k .
$$

Let

$$
T_{1}=T-\sum_{i=1}^{k} c_{i} A_{i}, \text { and } B_{j}=A_{j}-\sum_{i=1}^{k} a_{i j} A_{i} .
$$

Note $B_{j}=0$ for $j=1, \ldots, k$. By (6) and (7), we have

$$
\left(\begin{array}{c}
T_{1} x_{2} \\
\vdots \\
T_{1} x_{m}
\end{array}\right)=t_{k+1}\left(\begin{array}{c}
B_{k+1} x_{2} \\
\vdots \\
B_{k+1} x_{k}
\end{array}\right)+\ldots+t_{n}\left(\begin{array}{c}
B_{n} x_{2} \\
\vdots \\
B_{n} x_{k}
\end{array}\right) .
$$

Let $M=\operatorname{span}\left\{A_{1} x_{1}, \cdots, A_{k} x_{1}\right\}$ and $\widehat{\mathcal{S}}=\operatorname{span}\left\{B_{k+1}, \cdots, B_{n}\right\}$. By (3), $B_{j} x_{1}=$ 0 , for $j=1, \ldots, n$. Thus, by Lemma $4, R\left(B_{j}\right) \subseteq M$, for $j=1, \ldots, n$, i.e. $\widehat{\mathcal{S}} \subseteq \mathcal{S}_{M}$.

If $\mathcal{S}=\mathcal{S}_{M}$, then $\mathcal{S}$ is $k$-reflexive by Lemma 6 .

If $\mathcal{S} \neq \mathcal{S}_{M}$, then Lemma 3 implies $k\left(\mathcal{S}_{M}\right) \leq k-1$. Thus $k(\widehat{\mathcal{S}}) \leq k-1$. By equation (8), $T_{1}^{(k-1)} \in \operatorname{ref} f_{a}\left(\widehat{\mathcal{S}}^{(k-1)}\right)$. By the induction hypothesis, $\widehat{\mathcal{S}}$ is algebraically $(k-1)$-reflexive; thus, $T_{1} \in \widehat{\mathcal{S}} \subseteq \mathcal{S}$. Therefore $T \in \mathcal{S}$.

Proof of Theorem 1. If $n=1$ or 2, the conclusion follows from Lemma 7. Suppose the result holds for $\operatorname{dim} \mathcal{S} \leq n-1, n \geq 3$. Let $\operatorname{dim} \mathcal{S}=n, k(\mathcal{S})=k$, and $j=\lfloor\sqrt{2 n}\rfloor$. If $k \leq j$, by Lemma $7, \mathcal{S}$ is algebraically $k$-reflexive, thus algebraically $j$-reflexive.

Suppose $k>j$. If $k=n$, then $\mathcal{S}$ is algebraically 2-reflexive. Hence $\mathcal{S}$ is algebraically $j$-reflexive. Suppose $j<k \leq n-1$. Since $j=\lfloor\sqrt{2 n}\rfloor$, it follows that $2 n<(j+1)^{2}$. Thus, $2(n-k)<(j+1)^{2}-2 k \leq(j+1)^{2}-2(j+1)=j^{2}-1$. Hence, $\lfloor\sqrt{2(n-k)}\rfloor \leq j-1$. Using the same argument as that of Lemma 7 , we can obtain 
an equation similar to (8). Note that $\operatorname{dim}\left(\operatorname{span}\left\{B_{k+1}, \ldots, B_{n}\right\}\right) \leq n-k$; by the induction hypothesis, $\operatorname{span}\left\{B_{k+1}, \ldots, B_{n}\right\}$ is algebraically $\lfloor\sqrt{2(n-k)}\rfloor$-reflexive since $j-1 \geq\lfloor\sqrt{2(n-k)}\rfloor$. Thus $\operatorname{span}\left\{B_{k+1}, \ldots, B_{n}\right\}$ is algebraically $(j-1)$-reflexive, so $\mathcal{S}$ is algebraically $j$-reflexive.

\section{REFERENCES}

1. E. Azoff, On finite rank operators and preannihilators, Memoir Amer. Math. Soc. 357 (1986). MR 0858467 (88a:47041)

2. Lifeng Ding, On a pattern of reflexive operator spaces, Proc. Amer. Math. Soc. (10) 124 (1996), 3101-3108. MR1343689 (97h:47039)

3. Don Hadwin, A general view of reflexivity, Trans. Amer. Math. Soc. 344 (1994), 325-360. MR.1239639 (95f:47071)

4. Don Hadwin, Algebraically reflexive linear transformations, Linear and Multilinear Algebra (3) 14 (1983), 225-233. MR0718951 (85e:47003)

5. D. R. Larson, Reflexivity, algebraic reflexivity and linear interpolation, Amer. J. Math. 110 (1988), 283-299. MR0935008 (89d:47096)

6. J. Li and Z. Pan, Reflexivity of finite-dimensional subspaces of operators, J. Oper. Theory. 46 (2001), 381-389. MR.1870413 (2002j:47119)

7. B. Magajna, On the relative reflexivity of finitely generated modules of operators, Trans. Amer. Math. Soc. 327 (1991), 221-249. MR1038017 (91m:47064)

Department of Mathematics, East China University of Science and Technology, Shanghai 200237, People's Republic of China

E-mail address: jiankuili@yahoo.com

Department of Mathematical Sciences, Saginaw Valley State University, University Center, Michigan 48710

E-mail address: pan@svsu.edu 